\title{
Qubits from Black Holes in M-theory on K3 Surface
}

\author{
Adil Belhaj ${ }^{1}$, Zakariae Benslimane ${ }^{2}$, Moulay Brahim Sedra ${ }^{2}$, Antonio Segui ${ }^{3}$ \\ ${ }^{1}$ LIRST, Département de Physique, Faculté Polydisciplinaire, Université Sultan Moulay Slimane \\ Béni Mellal, Morocco \\ ${ }^{2}$ Département de Physique, LabSIMO, Faculté des Sciences, Université Ibn Tofail \\ Kénitra, Morocco \\ ${ }^{3}$ Departamento de Física Teórica, Universidad de Zaragoza, E-50 009-Zaragoza, Spain
}

\begin{abstract}
Using M-theory compactification, we develop a three factor separation for the scalar submanifold of $N=2$ seven dimensional supergravity associated with 2-cycles of the K3 surface. Concretely, we give an interplay between the three scalar submanifold factors and the extremal black holes obtained from M2-branes wrapping such 2-cycles. Then, we show that the corresponding black hole charges are linked to one, two and four qubit systems.
\end{abstract}

Keywords: Qubit systems, black holes, M-theory and K3 surface. 


\section{Introduction}

Extremal black holes have been extensively investigated in the context of string theory and related topics including $\mathrm{M}$ and $\mathrm{F}$ theories [1, 2, 3]. These objects are obtained from branes wrapping nontrivial cycles in the Calabi-Yau manifolds. The corresponding scalar moduli can be fixed in terms of the black brane charges using the attractor mechanism [4, 5, 6]. The minimum of the effective potential generates fixed values of the stringy scalar fields. This issue has been also connected with quantum information based on the qubit formalism [7, 8]. In fact, a nice mapping between eight charges of STU black holes and three qubits have been reported in [9]. This link has been derived from type IIB superstring compactification on a six-torus $T^{6}$ using string duality. Alternative methods based on graph theory using Adinkras and toric geometry have been developed to support such a correspondence [10, 11, 12. More precisely, an Adinkra graphic representation of the extremal black branes constructed from the toroidal compactification of type IIA superstring on $T^{n}$ have been proposed in [10. This analysis has been generalized to $n$-superqubits. Concretely, it has been shown that the number of bosonic

and fermionic states are $\frac{3^{n}+1}{2}$ and $\frac{3^{n}-1}{2}$, associated with odd and even geometries on the real supermanifold $T^{n \mid n}$, respectively .

The aim of this work is to further extend these works by going beyond the toroidal compactification in type II superstrings. More precisely, we reconsider the study of the moduli space of M-theory on the K3 surface as a leading example of Calabi-Yan manifolds. This analysis offers a new take on the moduli subspace of 2-cycles on which M2-branes are wrapped to produce seven dimensional black holes. The focus is on such geometries which in turn can potentially be interpreted as the moduli space of three classes of black holes. In particular, consider in some detail such classes, we find that they are linked to one, two and four qubits systems.

The organization of this paper is as follows. In section 2, we develop a new three factor separation for the scalar submanifold of $N=2$ seven dimensional supergravity associated with 2-cycles of the K3 surface on which M-theory is compactified. The identification of each factor is based on the appearance of three different real 2-forms of the K3 surface appearing in the untwisted and the twisted sectors. In section 3, we point out the existence of a link between the extremal black hole charges and qubit systems using the compactification of M-theory on the K3 surface. The last section is devoted to discussions and open questions.

\section{On the moduli space of black holes in M-theory on the K3 surface}

In this section, we reconsider the analysis of the moduli space of $N=2$ supergravity in seven dimensions arising from the M-theory on the K3 surface [13, 14]. The embedding of 
extremal black holes in such a compactification provides a new factorization scheme for the scalar submanifolds associated with real 2-forms of the K3 surface. This decomposition will be used to establish a mapping with lower dimensional qubits corresponding to the untwisted and twisted sectors. We begin by, briefly, recalling that the K3 surface is a 2-dimensional Calabi-Yau manifold with a Kähler structure permitting the existence of a global nonvanishing holomorphic 2-form. Equivalently, it can be defined as a Kähler manifold with a vanishing first Chern class $c_{1}=0$ and $\mathbf{S U}(2)$ Holonomy group [15]. It is noted that K3 involves a Hodge diagram playing a crucial role in the determination of the stringy spectrum in lower dimensions [16]. The string, M, or F, theory compactification on K3 preserves only half of the initial supercharges. A close inspection shows that one can construct such a manifold using different ways, including physical methods based on supersymmetric linear sigma models in two dimensions. The famous one concerns the orbifold construction which is given by $T^{4}$ modulo discrete isometries of $\mathbf{S U}(2)$. Concretely, we take the 4-torus $T^{4}$ parameterized by four real coordinates $x_{i},(i=1, \ldots, 4)$, subject to the following identifications

$$
x_{i}=x_{i}+1
$$

It is convenient to use the complex coordinates with the following identification constraints

$$
z_{i}=z_{i}+1, \quad z_{i}=z_{i}+\imath, \quad i=1,2
$$

For simplicity reason, we consider the $\mathbb{Z}_{2}$ symmetry [15]. In this way, the orbifold $T^{4} / \mathbb{Z}_{2}$ is obtained from $T^{4}$ by imposing an extra $\mathbb{Z}_{2}$ symmetry acting on the complex variables $z_{i}$ as follows

$$
z_{i} \rightarrow-z_{i}
$$

To study the cohomology classes of the K3 surface, we look for the forms on $T^{4}$ which are invariant under such a symmetry. Indeed, these invariant forms belonging to the untwisted sector are given by

$$
1, \quad d z_{1} \wedge d z_{2}, \quad \overline{d z}_{1} \wedge \overline{d z}_{2}, \quad \overline{d z}_{i} \wedge d z_{j}, \quad d z_{1} \wedge d z_{2} \wedge \overline{d z}_{1} \wedge \overline{d z}_{2}
$$

\begin{tabular}{|c|c|c|c|c|}
\hline \multirow[b]{3}{*}{$h^{2,0}$} & $h^{0,0}$ & & \multicolumn{2}{|c|}{1} \\
\hline & $h^{0,1}$ & & 0 & 0 \\
\hline & $h^{1,1}$ & $h^{0,2}=1$ & & \\
\hline$h^{2,1}$ & $h^{1,2}$ & & 0 & 0 \\
\hline & $h^{2,2}$ & & & \\
\hline
\end{tabular}

It is recalled that $h^{p, q}$ denotes the number of the holomorphic and the anti-holomorphic forms of degree $(p, q)$. These numbers are listed in the following Hodge diagram 
It has been shown that there are 16 fixed points which read as

$$
\left(z_{1}^{i}, z_{2}^{i}\right)=(0,0),\left(0, \frac{1}{2}\right),\left(0, \frac{1}{2} \imath\right),\left(0, \frac{1}{2}+\frac{1}{2} \imath\right) \ldots\left(\frac{1}{2}+\frac{1}{2} \imath, \frac{1}{2}+\frac{1}{2} \imath\right)
$$

modifying the Hodge number $h^{1,1}$. The blowing up of all 16 fixed points produces the so-called K3 surface. Each fixed point corresponds to a vanishing 2-sphere.

Let us explain how to blow up a singularity. Locally, the orbifold $T^{4} / Z_{2}$ looks like $\mathbb{C}^{2} / \mathbb{Z}_{2}$ which is known by $A_{1}$ space described by the following equation $x y=z^{2}$ where $x, y$ and $z$ are $Z_{2}$-invariant. This can be related to the coordinates of $\mathbb{C}^{2}$ as follows $z=z_{1} z_{2}, x=z_{1}^{2}, y=z_{2}^{2}$. This local geometry involves a singularity at $x=y=z=0$ which can be replaced by a two dimensional sphere $S^{2}$ which is isomorphic to $\mathbb{C} P^{1}$. The blowing up of 16 fixed points changes $h^{1,1}=4$ which becomes $h^{1,1}=4+16=20$. The Hodge diagram, involving the untwisted and twisted sectors, takes the following form

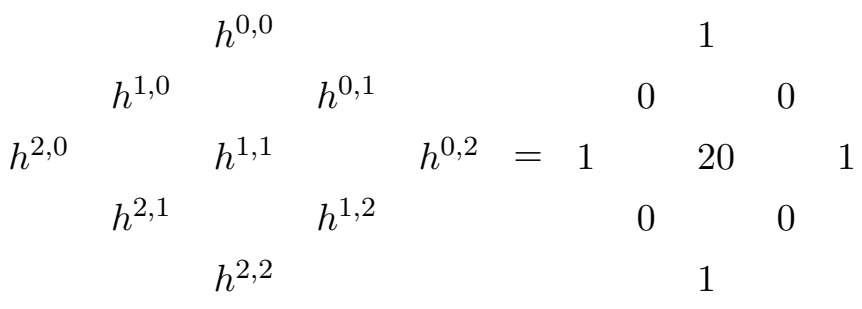

Many geometrical properties of the compactification on the K3 surface can be obtained from this simple diagram. The central horizontal line gives information on the moduli of the K3 surface, associated with 2-cycles, controlled by the parameters of the complex and the Kähler deformations. It is recalled that the K3 surface is a self-mirror manifold. It turns out that the Hodge diagram carries not only geometric but also physical data of the compactified theories. The relevant physical information, including the black holes, can be obtained from the second real cohomology $H^{2}(K 3, \mathbb{R})$ of the $\mathrm{K} 3$ surface which can be decomposed as follows

$$
H^{2}(K 3, \mathbb{R})=H^{2,0}(K 3, \mathbb{R}) \oplus H^{1,1}(K 3, \mathbb{R}) \oplus H^{0,2}(K 3, \mathbb{R}) .
$$

Its dimensions, as a vector space, is

$$
\operatorname{dim} H^{2}(K 3, \mathbb{R})=\operatorname{dim} H^{2,0}(K 3, \mathbb{R})+\operatorname{dim} H^{1,1}(K 3, \mathbb{R})+\operatorname{dim} H^{0,2}(K 3, \mathbb{R})=22 .
$$

The compactification of M-theory on the K3 surface gives $N=2$ supergravity in seven dimensions [13]. It is recalled that, at low energy limit, the eleven dimensional bosonic massless fields of M-theory are

$$
g_{M N}, C_{M N K}, \quad M, N, K=0, \ldots, 10
$$

which are the metric and the 3 -form gauge potential respectively. The dimensional reduction on the K3 surface produces the following seven dimensional fields

$$
g_{\mu \nu}, C_{\mu \nu \rho}, C_{\mu i j}, \quad \phi_{a}, \quad \mu, \nu=0, \ldots, 6, \quad a=1, \ldots, 58
$$


where $g_{\mu \nu}$ is the seven dimensional metric, $C_{\mu i j}$ are Maxwell gauge fields arising from the compactification of $C_{\mu \nu \rho}$ on the real 2-cycles of the K3 surface. The total moduli space of M-theory on the K3 surface, parameterized by $\phi_{a}$, reads as

$$
M=\frac{\mathbf{S O}(3,19)}{\mathbf{S O}(3) \times \mathbf{S O}(19)} \times S O(1,1) .
$$

In the study of the extremal black objets in M-theory compactification on the K3 surface, four seven dimensional black $p$-brane solutions can appear as required by the dual condition [17]

$$
p+q=3
$$

They are defined by $p=0,1,2,3$ with $A d S_{p+2} \times S^{5-p}$ near-horizon geometries. These solutions are classified by

\begin{tabular}{|c|c|c|}
\hline$p$ & Black object & near-horizon geometry \\
\hline$p=0$ & Black holes & $A d s_{2} \times S^{5}$ \\
\hline$p=1$ & Black M-string & $A d s_{3} \times S^{4}$ \\
\hline$p=2$ & Black M2-brane & $A d s_{4} \times S^{3}$ \\
\hline$p=3$ & Black M3-branes & $A d s_{5} \times S^{2}$ \\
\hline
\end{tabular}

Table 1: This table gives the possible extremal black $p$-brane solutions in M-theory on K3 surface.

In fact, $p=0$ corresponds to an $A d S_{2} \times S^{5}$ space describing the near-horizon geometry of the electric charged black holes. They are obtained from M2-branes wrapping 2-cycles in the K3 surface. Their magnetic dual are black M3-branes with $A d S_{5} \times S^{2}$ near-horizon geometries, which are obtained from M5-branes wrapping also 2-cycles. The objects carry charges associated with the gauge invariant field strengths $F^{i}=d A^{i}(i=1, \ldots, 22)$ of the $N=2$ supergravity theory derived from $C_{\mu \nu \rho}$ on the real 2-cycles of the K3 surface. However, $p=1$ corresponds to the $A d S_{3} \times S^{4}$ space describing the near-horizon geometry of an extremal black string. It can be obtained from a M5-brane wrapping the K3 surface. It is charged under the 3-form field strengths obtained from the compactification of the K3 surface. The electric charge is proportional to the integral of the dual of $H=d C$ over a 3-cycle. The magnetic dual horizon geometry reads as $A d S_{4} \times S^{3}$ and it describes a black M2-brane. The latter is charged under the gauge invariant 4-form field strengths $H$.

Combining the Hodge diagram and the moduli space information of the K3 surface, we can establish a mapping between the black object charges and the moduli space of the K3 surface. A priori, there are many ways to approach such a space depending on addressed questions [17. In fact, it is recalled that the moduli space $\mathcal{M}$ can be associated with the forms on the K3 
surface. Indeed, the first factor $\frac{\mathbf{S O}(3,19)}{\mathbf{S O}(3) \times \mathbf{S O}(19)}$ is associated with 22 real 2 -forms

$$
\frac{\mathbf{S O}(3,19)}{\mathbf{S O}(3) \times \mathbf{S O}(19)} \rightarrow\{2 \text {-forms }\}
$$

However, the remaining factor corresponds to the 0-form and its dual 4-form

$$
\mathbf{S O}(1,1) \rightarrow\{0 \text {-form, dual 4-form }\}
$$

This factorization is directly related to the classification of the black object charges in seven dimensions obtained from M2 and M5-branes discussed above. In fact, we have the following mapping

$$
\begin{aligned}
\frac{\mathbf{S O}(3,19)}{\mathbf{S O}(3) \times \mathbf{S O}(19)} & \rightarrow\{p=0, p=3\} \\
\mathbf{S O}(1,1) & \rightarrow\{p=1, p=2\}
\end{aligned}
$$

The study of the attractor horizon geometries of extremal black $p$-branes on the $K 3$ surface can be explored to get the complete correspondence

\begin{tabular}{|c|c|c|c|}
\hline Coset space & Black brane & Cycles in K3 & Gauge symmetry \\
\hline$\frac{\mathbf{S O}(3,19)}{\mathbf{S O}(3) \times \mathbf{S O}(16)}$ & black holes (black M3-branes) & 2-cycles & $\mathbf{U}(1)^{22}$ \\
\hline $\mathbf{S O}(1,1)$ & Black M-string (black M2-brane) & K3 (0-cycle) & $\mathbf{U}(1)$ \\
\hline
\end{tabular}

Table 2: This table gives the correspondence between the scalar manifold factors and extremal black $p$-brane charges in M-theory on the K3 surface.

\section{$3 \quad$ Embedding qubits in M-theory black holes}

In this section, we will show that qubits can be embedded in M-theory on the K3 surface. Concretely, we would like to elaborate a link between black holes and the moduli subspace $\frac{\mathbf{S O}(3,19)}{\mathbf{S O}(3) \times \mathbf{S O}(19)}$. This correspondence will be explored to engineer qubit systems from the corresponding black holes (black M3-branes). It is worth to recall that the qubit is a primordial building block in quantum version of information theory [18, 19, 20]. This piece describes a system with two physical states. Using Dirac notation, a single qubit reads as

$$
|\psi\rangle=c_{0}|0\rangle+c_{1}|1\rangle
$$

where $c_{i}$ are complex numbers verifying the normalization condition

$$
\left|c_{0}\right|^{2}+\left|c_{1}\right|^{2}=1 .
$$


Geometrically, this probability condition generates a two-sphere called Bloch sphere. Similarly, $\mathrm{T}$ the two qubits are four state systems taking the following form

$$
|\psi\rangle=c_{00}|00\rangle+c_{10}|10\rangle+c_{01}|01\rangle+c_{11}|11\rangle
$$

where $c_{i j}$ are complex coefficients satisfying the normalization condition

$$
\left|c_{00}\right|^{2}+\left|c_{10}\right|^{2}+\left|c_{01}\right|^{2}+\left|c_{11}\right|^{2}=1
$$

This equation describes a three dimensional complex projective space $\mathbb{C} P^{3}$ extending the Bloch sphere. This analysis can be generalized to $n$ qubits associated with $2^{n}$ state systems placed on $\mathbb{C} P^{2^{n}-1}$ state geometry. An inspection shows that there is a direct correspondence between qubits and black brane charge vectors in M-theory on the K3 surface. These black solutions, associated with $p=0$ and $p=3$, can be obtained from M2 and M5-branes wrapping 2-cycles in the K3 surface respectively. To get such a connection, we shall reconsider the study of the second cohomology space $H^{2}(K 3, \mathbb{R})$ of the $K 3$ surface. In particular, we shall use a special factorization of the corresponding moduli space. The orbifold construction can be worked out to show that the moduli subspace of M-theory on K3 surface should have a prior three factors. Under this hypothesis, the scalar submanifold $\frac{\mathbf{S O}(3,19)}{\mathbf{S O}(3) \times \mathbf{S O}(19)}$ associated with 2 -forms should take the form

$$
\frac{\mathbf{S O}(3,19)}{\mathbf{S O}(3) \times \mathbf{S O}(19)}=M_{1} \times M_{2} \times M_{3} .
$$

We will show that this factorization can be related to the existence of three different classes of 2-cycles embedded in the K3 surface. The identification of each factor can be obtained by the help of the the second real cohomology $H^{2}(K 3, \mathbb{R})$. Indeed, the submanifold group $\frac{\mathbf{S O}(3,19)}{\mathbf{S O}(3) \times \mathbf{S O}(19)}$ can be factorized as follows

$$
\frac{\mathbf{S O}(3,19)}{\mathbf{S O}(3) \times \mathbf{S O}(19)}=\frac{\mathbf{S O}(3,3)}{\mathbf{S O}(3) \times \mathbf{S O}(3)} \times \frac{\mathbf{S O}(3,19)}{\mathbf{S O}(3) \times \mathbf{S O}(19)}
$$

The submanifold $\frac{\mathbf{S O}(3,3)}{\mathbf{S O}(3) \times \mathbf{S O}(3)}$ carries information on the untwisted sector associated with the former Hodge diagram. The second factor corresponds to the twisted sector controlled by the 16 fixed points of the orbifold construction. To embed lower dimensional qubits, we need to use an extra factorization. In fact, it is given by

$$
\frac{\mathbf{S O}(3,19)}{\mathbf{S O}(3) \times \mathbf{S O}(19)}=\frac{\mathbf{S O}(3,2)}{\mathbf{S O}(3) \times \mathbf{S O}(2)} \times \frac{\mathbf{S O}(3,1)}{\mathbf{S O}(3)} \times \frac{\mathbf{S O}(3,16)}{\mathbf{S O}(3) \times \mathbf{S O}(16)} .
$$

Then, we decompose the untwisted sector to

$$
\frac{\mathbf{S O}(3,2)}{\mathbf{S O}(3) \times \mathbf{S O}(2)} \times \frac{\mathbf{S O}(3,1)}{\mathbf{S O}(3)} \rightarrow \frac{\mathbf{S O}(2,2)}{\mathbf{S O}(2) \times \mathbf{S O}(2)} \times \frac{\mathbf{S O}(2,1)}{\mathbf{S O}(2)} .
$$

The factor $\frac{\mathbf{S O}(2,2)}{\mathbf{S O}(2) \times \mathbf{S O}(2)}$ can be linked with the 2-forms $\overline{d z}_{i} \wedge d z_{j}$ belonging to the untwisted sector $H^{1,1}(K 3, \mathbb{R})$. However, the factor $\frac{S O(2,1)}{S O(2)}$ will be related with the real parts of the complex holomorphic and antiholomorphic 2-forms which are elements of the cohomology classes 
$H^{2,0}(K 3, \mathbb{R})$ and $H^{2,0}(K 3, \mathbb{R})$ respectively. The twisted sector $\frac{\mathbf{S O}(3,16)}{\mathbf{S O}(3) \times \mathbf{S O}(16)}$ should be factorized as follows

$$
\frac{\mathbf{S O}(3,16)}{\mathbf{S O}(3) \times \mathbf{S O}(16)}=\frac{\mathbf{S O}(1,16)}{\mathbf{S O}(16)} \times \frac{\mathbf{S O}(2,16)}{\mathbf{S O}(2) \times \mathbf{S O}(16)} .
$$

The factor $\frac{\mathbf{S O}(1,16)}{\mathbf{S O}(16)}$ can be associated with the 2-cycles used in the deformation of the 16 fixed points. However, the factor $\frac{\mathbf{S O}(2,16)}{\mathbf{S O}(2) \times \mathbf{S O}(16)}$ carries information of the quaternionic structure of the K3 surface which has no role in the present discussion. This factor will be omitted. Finally, we consider the following decomposition

$$
\frac{\mathbf{S O}(3,19)}{\mathbf{S O}(3) \times \mathbf{S O}(19)} \rightarrow \frac{\mathbf{S O}(2,1)}{\mathbf{S O}(2)} \times \frac{\mathbf{S O}(2,2)}{\mathbf{S O}(2) \times \mathbf{S O}(2)} \times \frac{\mathbf{S O}(1,16)}{\mathbf{S O}(16)} .
$$

Analyzing these moduli space factors and M2-brane charges, the total abelian gauge group takes the following form

$$
\mathbf{U}(1)^{2} \times \mathbf{U}(1)^{4} \times \mathbf{U}(1)^{16}
$$

This separation of the charges is governed by the Hodge diagram encoding the K3 surface forms. It is suggested that $\mathbf{U}(1)^{2}$ can be associated with the coset space $\frac{\mathbf{S O}(2,1)}{\mathbf{S O}(2)}$. The second factor $U(1)^{4}$ is the abelian gauge symmetry associated with four field strength 2 -forms $F^{i j}(i, j=1,2)$. This $\mathbf{U}(1)^{4}$ gauge symmetry can be decomposed as follows

$$
\mathbf{U}(1)^{4}=\mathbf{U}(1)^{2} \times \mathbf{U}(1)^{2} .
$$

This part has $\mathbf{S O}(2) \times \mathbf{S O}(2)$ isotropy symmetry. The corresponding gauge fields are 1-forms obtained from the the reduction of the M-theory 3-form on 2-cycles belonging to the untwisted $H^{1,1}(K 3, \mathbb{R})$. The corresponding four charges can be related to the scalar submanifold factor $\frac{\mathbf{S O}(2,2)}{\mathbf{S O}(2) \times \mathbf{S O}(2)}$. The last gauge symmetry $\mathbf{U}(1)^{16}$ corresponds to strength 2-forms $F^{i}(i=1, \ldots, 16)$ which are obtained from the the reduction of the 3 -form gauge potential $C_{M N K}$ on 2-cycles used in the deformation of the singular points. They are elements of the twisted sector $H^{1,1}(K 3, R)$. Finally, we propose the following mapping

\begin{tabular}{|c|c|c|}
\hline Coset space & Black M-brane objects & Gauge symmetry \\
\hline$\frac{\mathbf{S O}(2,1)}{\mathbf{S O}(2)}$ & black holes (black M3-branes) & $\mathbf{U}(1)^{2}$ \\
\hline$\frac{\mathbf{S O}(2,2)}{\mathbf{S O}(2) \times \mathbf{S O}(2)}$ & black holes (black M3-branes) & $\mathbf{U}(1)^{4}$ \\
\hline$\frac{\mathbf{S O}(1,16)}{\mathbf{S O}(16)}$ & black holes (black M3-branes) & $\mathbf{U}(1)^{16}$ \\
\hline
\end{tabular}

Table 3: This table describes the relation between scalar submanifold factors, extremal black hole (brane) charges and the gauge symmetry in seven dimensions.

Form the orbifold construction point of view, it is remarked that the seven dimensional gauge field can split into three parts

$$
A_{\mu}^{a}=A_{\mu}^{\alpha} \oplus A_{\mu}^{\beta} \oplus A_{\mu}^{\gamma}
$$


which are vectors in $\mathbf{S O}(2), \mathbf{S O}(2,2)$ and $\mathbf{S O}(16)$ symmetries respectively. This decomposition shows that 22 abelian vector fields can produce three classes of black hole charges depending on the 2-form type of the orbifold $\frac{T^{4}}{\mathbb{Z}_{2}}$. This classification allows one to embed lower dimensional qubits in such black holes obtained from a set of M2-branes wrapping the associated 2-cycles in the K3 surface.

\subsection{One qubit}

1-qubit can be embedded in the black hole physics in M-theory on the K3 surface which can be related to the scalar submanifold $\frac{\mathbf{S O}(2,1)}{\mathbf{S O}(2)}$ associated with $\mathbf{U}(1)^{2}$ gauge symmetry. The modeling of the 1-qubit can be done as in [9], using the holomorphic and antiholomorphic forms. The present qubit representation will be related to the $\frac{T^{4}}{\mathbb{Z}_{2}}$ holomorphic and antiholomorphic 2-forms which are elements of the cohomology classes $H^{2,0}$ and $H^{0,2}$ respectively

$$
\Omega=d z_{1} \wedge d z_{2}, \quad \bar{\Omega}=\overline{d z}_{1} \wedge \overline{d z}_{2}
$$

In order to establish the correspondence between these 2 -forms and the basis vectors of the one-qubit system, we can explore the scenario proposed in $[9]$. Indeed, it is recalled that for a $(p, q)$-form, the Hodge star action reads as

$$
(\varphi, \varphi) \frac{\omega^{2}}{2}=\varphi \wedge \star \bar{\varphi}
$$

where $\omega=\imath\left(\overline{d z_{1}} \wedge d z_{2}+\overline{d z_{1}} \wedge d z_{2}\right)$. It has been verified that for the orbifold $\frac{T^{4}}{Z_{2}}$, the Hodge star action reduces to

$$
(\varphi, \varphi) \frac{\omega^{2}}{2}=\varphi \wedge \bar{\varphi}
$$

Thus, the vector space calculation produces the following relations

$$
\begin{gathered}
(\Omega, \Omega)=(\bar{\Omega}, \bar{\Omega})=1 \\
(\Omega, \bar{\Omega})=(\bar{\Omega}, \Omega)=0 .
\end{gathered}
$$

This shows that $\{\Omega, \bar{\Omega}\}$ forms a two dimensional basis. Due to this calculation, we can take these vectors as a basis of 1-qubit. The mapping is given by

$$
\begin{aligned}
& \Omega \rightarrow|0\rangle \\
& \bar{\Omega} \rightarrow|1\rangle .
\end{aligned}
$$

The associated seven dimensional black hole has electric charges under $\mathbf{U}(1)^{2}$ gauge symmetry

$$
q_{0}, \quad q_{1}
$$

To get these charges, one uses the following real 2-forms

$$
\operatorname{Re}(\Omega)=\omega_{0}, \quad \operatorname{Im}(\bar{\Omega})=\omega_{1} .
$$


Indeed, each state $|i\rangle$ corresponds to M2-branes with charges $q_{i}$ which are given by

$$
q_{i}=\int_{C_{i}^{2}} F^{2}, \quad i=0,1,
$$

where $F^{2}=q^{\ell} w_{\ell}$ and where $C_{i}^{2}$ are 2-cycles dual to 2-forms $\omega_{i}$.

\subsection{Two qubits}

The 2-qubit can be also embedded in the untwisted sector. Concretely, we show that the $(1,1)$ forms $\omega_{i j}=\overline{d z_{i}} \wedge d z_{j},(i, j=1,2)$ can be worked out to form a basis of states describing 2-qubits. To get the corresponding basis of states, we need first to establish a symmetric bilinear scalar product. Indeed, we propose the following scalar product

$$
\left(\omega_{i j}, \omega_{k \ell}\right)=\int_{\frac{T^{4}}{\mathbb{Z}_{2}}} \omega_{i j} \wedge \perp \omega_{k \ell}
$$

where $\perp$ is an operator acting on $(1,1)$-forms $\omega_{i j}$ as follows

$$
\perp: \omega_{i j} \rightarrow \omega_{i+1 j+1} \quad(\bmod 2) .
$$

We will see that the orthogonality relations can be obtained by using the normalized volume form on the K3 surface

$$
\int_{\frac{T^{4}}{\mathbb{Z}_{2}}} \frac{w^{2}}{2}=1 .
$$

Using the above equations, we can show that

$$
\left(\omega_{i j}, \omega_{k \ell}\right)=\delta_{i k} \delta_{j \ell}
$$

In this way, the basis state of two qubits can be obtained from the following mapping $(1,1)$-forms of the untwisted sector

$$
\omega_{i j} \rightarrow|i-1 j-1\rangle, \quad i, j=1,2 .
$$

Combining these equations, we can write the following relations

$$
\left(\omega_{i j}, \omega_{k \ell}\right)=\langle i-1 j-1 \mid k-1 \ell-1\rangle=\delta_{i k} \delta_{j \ell} .
$$

In connection with seven dimensional black hole charges, each state $|i j\rangle$ corresponds to M2branes with charges $q^{i j}$ obtained by the following integration

$$
q^{i j}=\int_{C_{i j}^{2}} F^{2} .
$$

where $F^{2}=q^{k \ell} w_{k \ell}$.

Moreover, It is possible to recover the entangled states from a tensor representation of $(1,1)$ forms $\omega_{i j}$. The latter is given by

$$
\left[\omega_{i j}\right]=\left(\begin{array}{ll}
\omega_{11} & \omega_{12} \\
\omega_{21} & \omega_{22}
\end{array}\right)
$$


The trace of this tensor reads

$$
\text { Trace } \omega_{i j}=\omega_{11}+\omega_{22} \text {. }
$$

Using the normalization condition, this trace produces the following Bell state

$$
\sqrt{2} \text { Trace } \omega_{i j} \rightarrow|00\rangle+|11\rangle \text {. }
$$

It is recalled that the antitrace can be written as

$$
\text { Antitrace } \omega_{i j}=\omega_{21}-\omega_{12} \text {. }
$$

Similarly, we get the following state

$$
\sqrt{2} \text { Antitrace } \omega_{i j} \rightarrow|10\rangle-|01\rangle \text {. }
$$

The last comment that we should make on the above tensor concerns its determinant. By replacing the usual product by wedge multiplication, it reads as

$$
\text { Det } \omega_{i j}=\omega_{11} \wedge \omega_{22}-\omega_{21} \wedge \omega_{12}
$$

Integrating this form on the K3 surface, we get the following constraint on the black holes charges

$$
q_{11} q_{22}=q_{21} q_{12}
$$

It seems that there could be a link with entanglement and Segre embedding discussed in connection with conifold geometries in type II superstrings [21]. It is recalled that the Segre embedding is considered as a mapping taking products of projective Hilbert spaces as a projective variety. In fact, it is defined as follows

$$
\sigma: P^{n} \times P^{m} \rightarrow \Sigma_{n, m} \equiv P^{(n+1)(m+1)-1}
$$

where $P^{n}, P^{m}$ are projective Hilbert spaces and $\Sigma_{n, m}$ is known as the Segre variety. We will give a concise description for the physical case of two fermionic systems, namely the case $m=n=1$ [21]. Higher dimensional cases are straightforward.

To establish the link with entanglement, let us consider two non-interacting fermionic systems. The corresponding Hilbert space reads as $\mathbb{C}^{2} \otimes \mathbb{C}^{2}=\mathbb{C}^{4}$. The normalization constraint implies that the space of states is $\mathbb{C} P^{3}$. Each system has a state representation in terms of the Bloch sphere $S^{2}=\mathbb{C} P^{1}$. Thus, a general state $|\Psi\rangle$ can be represented by $\mathbb{C} P^{1} \otimes \mathbb{C} P^{1}$ as follows

$$
|\Psi\rangle=\left|\Psi_{1}\right\rangle \otimes\left|\Psi_{2}\right\rangle
$$

where $\left|\Psi_{1}\right\rangle=\alpha_{1}|+\rangle+\beta_{1}|-\rangle$ and where $\left|\Psi_{2}\right\rangle=\alpha_{2}|+\rangle+\beta_{2}|-\rangle$. This gives

$$
|\Psi\rangle=\alpha_{1} \alpha_{2}|++\rangle+\alpha_{1} \beta_{2}|+-\rangle+\alpha_{2} \beta_{1}|-+\rangle+\beta_{1} \beta_{2}|--\rangle .
$$


By rearranging the complex amplitudes in a 4-tuple of projective coordinates

$$
\left(z_{1}, z_{2}, z_{3}, z_{0}\right) \equiv\left(\alpha_{1} \alpha_{2}, \alpha_{1} \beta_{2}, \alpha_{2} \beta_{1}, \beta_{1} \beta_{2}\right),
$$

we get an hypersurface embedded in $\mathbb{C} P^{3}$ given by the non linear constraint

$$
z_{1} z_{0}=z_{2} z_{3} \quad \text { or } \quad \zeta_{1}=\zeta_{2} \zeta_{3}
$$

where $\left(\zeta_{1}, \zeta_{2}, \zeta_{3}\right) \equiv\left(z_{1} / z_{0}, z_{2} / z_{0}, z_{3} / z_{0}\right)$ are inhomogeneous coordinates on $\mathbb{C} P^{3}$. This constraint transforms the Kähler potential of $\mathbb{C} P^{3}$

$$
K=\log \left(1+\left|\zeta_{1}\right|^{2}+\left|\zeta_{2}\right|^{2}+\left|\zeta_{3}\right|^{2}\right)
$$

to

$$
K=\log \left(1+\left|\zeta_{2}\right|^{2}\right)+\log \left(1+\left|\zeta_{3}\right|^{2}\right)
$$

describing the product of Fubini-Study metrics on $\mathbb{C} P^{1} \times \mathbb{C} P^{1}$. Thus, from a superposition of separable states $\mathbb{C} P^{1} \times \mathbb{C} P^{1}$, we get entangled states by the Segre embedding into $\mathbb{C} P^{3}$. It turns out that $\mathbb{C} P^{1} \times \mathbb{C} P^{1}$ can be considered as a compact part of a local Calabi-Yau threefold. The manifold, which has been considered as a small resolution of conifold singularity, has been used for computing exact degeneracies of BPS black holes in type IIA superstring from D-branes 22]. Thinking about the black hole/ qubit correspondence, it is possible to make contact with the work presented here. We hope to come back to this issue in future.

It is also noted that the superoperators discussed in [23] can be related to the 2-qubit associated with the $\omega_{i j}$ forms. Interpreting the corresponding Hilbert space as a Liouvile space, a generalization in terms of a product of the K3 surface can be elaborated to discuss quantum four valued logic states. We will address this question in future works.

\subsection{Four qubits}

In this section, we treat the black hole charges obtained from the twisted sector. This can be related with 4-qubit states. To do so, we consider the second homology class associated with the deformation of the fixed points $\left(z_{1}^{i}, z_{2}^{i}\right)$ of the $\mathbb{Z}_{2}$ symmetry. It is recalled that, in the resolved geometry, each fixed point is replaced by a 2 -cycle

$$
\left(z_{1}^{i}, z_{2}^{i}\right) \rightarrow C_{2}^{i}, \quad i=1, \ldots, 16
$$

Up to a normalization, the 2-cycles are dual to real 2-forms $\omega_{i}$ such that

$$
\int_{C_{2}^{i}} w_{j}=\delta_{i j}
$$

To connect this with a 4-qubit system, we first need to construct the scalar product $\left(w_{i}, w_{j}\right)$ producing the orthogonality relations. To establish the corresponding formulae, one may explore certain geometric data of the K3 surface. It is worth noting that, in the K3 surface, 
there is a symmetric scalar quantity describing the intersection of 2-cycles. This information will be explored to work out the scalar product that we are after. Generally for ALE spaces, the singularity deformation consists on replacing a fixed point by a collection of intersecting 2-cycles according to the ADE Dynkin diagrams. Concretely, the intersection matrix of the 2-cycles used for the resolution of ADE singularities is, up to some details, the opposite of the ADE Cartan matrices $K_{i j}$ [24]. This eventually leads to a correspondence between the ADE roots and the 2-cycles. More specifically, to each simple root $\alpha_{i}$ we associate a single 2-cycle identified with the complex projective space $\mathbb{C P}^{1}$. Inspired by the intersection theory of the K3 surface, we can identify $\left(w_{i}, w_{j}\right)$ with the Cartan matrix

$$
\left(w_{i}, w_{j}\right) \equiv C_{2}^{i} \cdot C_{2}^{j}=-K_{i j} .
$$

It is recalled that the intersection matrix of $n$-dimensional sphere $S^{n}$, used in the blowups of singularities, is symmetric for $n$ even and antisymmetric for $n$ odd. In the case of the K3 surface, the matrix is symmetric showing that the inner product is symmetric and bilinear. This describes a particular case of hermitian inner product used in the quantum mechanics.

Roughly speaking, the natural of the singularity of the orbifold $\frac{T^{4}}{\mathbb{Z}_{2}}$ shows that the associated algebra is $\oplus_{i=1}^{16} \mathbf{s u}(2)$ Lie algebra. In this way, the quantity $\left(w_{i}, w_{j}\right)$ reads as

$$
\left(w_{i}, w_{j}\right)=-2 \delta_{i j}
$$

To complete the analysis, we can use the Wick rotation and the following scale transformation

$$
w_{i} \rightarrow \frac{\imath}{\sqrt{2}} w_{i}
$$

The 4-qubit basis of states can be obtained by using an appropriate correspondence. Indeed, to get a mapping, we associated to each fixed point $\left(z_{1}, z_{2}\right)$ a state. Using the cartesian coordinates $z_{1}=x_{1}+\imath x_{2}$ and $z_{2}=x_{3}+\imath x_{4}$, we can propose the following mapping

$$
\left(z_{1}, z_{2}\right) \rightarrow\left|2 x_{1} 2 x_{2} 2 x_{3} 2 x_{4}\right\rangle .
$$

representing a 2-cycle belonging to the twisted sector. Using these conventions, the basis states are given by

$$
(0,0) \rightarrow|0000\rangle, \quad\left(0, \frac{1}{2}\right) \rightarrow|0010\rangle, \quad \ldots \quad, \quad\left(\frac{1}{2}+\frac{1}{2} \imath, \frac{1}{2}+\frac{1}{2} \imath\right) \rightarrow|1111\rangle .
$$

Clearly, we have 16 quantized charges associated with M2-branes wrapping on $C_{2}^{z_{1}, z_{2}}$ associated with the fixed points $\left(z_{1}, z_{2}\right)$. Using a novel notation associated with such fixed points, these charges take the following matrix form

$$
q_{z_{1} z_{2}}=\left(\begin{array}{cccc}
q_{00} & q_{0 \frac{1}{2}} & q_{0 \frac{1}{2} \imath} & q_{0 \frac{1}{2}+\frac{1}{2} \imath} \\
q_{\frac{1}{2} 0} & q_{\frac{1}{2} \frac{1}{2}} & q_{\frac{1}{2} \frac{1}{2} \imath} & q_{\frac{1}{2} \frac{1}{2}+\frac{1}{2} \imath} \\
q_{\frac{1}{2} \imath 0} & q_{\frac{1}{2} \imath \frac{1}{2}} & q_{\frac{1}{2} \imath \frac{1}{2} \imath} & q_{\frac{1}{2} \imath \frac{1}{2}+\frac{1}{2} \imath} \\
q_{\frac{1}{2}+\frac{1}{2} \imath 0} & q_{\frac{1}{2}+\frac{1}{2} \imath \frac{1}{2}} & q_{\frac{1}{2}+\frac{1}{2} \imath \frac{1}{2} \imath} & q_{\frac{1}{2}+\frac{1}{2} \imath \frac{1}{2}+\frac{1}{2} \imath}
\end{array}\right) .
$$


They can be computed by the following integration

$$
q_{z_{1} z_{2}}=\int_{C_{2}^{z_{1} z_{2}}} F^{2}
$$

where $F^{2}$ can be decomposed in terms of the corresponding 2 -forms $w_{z_{1} z_{2}}$.

A further check shows that there are many geometric transformations acting of these states which may play the role of gates considered as qubit operators. In particular, a new $\mathbb{Z}_{2}$ symmetry can be implemented which acts as follows

$$
x \rightarrow x+\frac{1}{2}
$$

This transformation is quite different to the one used before. It has no fixed points associated with M2 and M5-brane configurations producing new black hole charges. It is found that there are 15 operations

$$
\sum_{k=1}^{4} C_{4}^{k}=15
$$

For instance, the operation acting as

$$
x_{i} \rightarrow x_{i}+\frac{1}{2}, \quad i=1, \ldots, 4
$$

produces the following 4-qubit operator

$$
\left|2 x_{1} 2 x_{2} 2 x_{3} 2 x_{4}\right\rangle \rightarrow\left|2 x_{1}+12 x_{2}+12 x_{3}+12 x_{4}+1\right\rangle \quad(\bmod 2) .
$$

It has been checked that this operation can be understood as a 4-flip bit.

\section{Conclusions and open questions}

We have reconsidered the analysis of the moduli subspace of $N=2$ seven dimensional supergravity obtained from M-theory on the K3 surface. Inspired by the Hodge diagram of the K3 surface, we have proposed a new three factor realization for the scalar submanifold of $N=2 \mathrm{su}-$ pergravity associated with the 2-cycles in the M-theory compactifications. The decomposition is based on the existence of three different 2-forms of the K3 surface. In particular, we have pointed out a correspondence between the scalar submanifold factors and the extremal black hole charges. We have specially focused on these factors and their relations to qubit systems. More precisely, we have considered in some details such black holes classes, and we have found that they are linked to one, two and four qubits systems.

It is observed that the analysis presented here might be extended to the case of F-theory on the K3 surface. This compactification provides an eight dimensional gauge theory [25, 26, 27, 
28. The F-theory construction requires that the K3 surface should involve an elliptic fibration structure. It is known that its moduli space reads as

$$
M=\frac{\mathbf{S O}(2,18)}{\mathbf{S O}(2) \times \mathbf{S O}(18)} \times \mathbb{R}^{+} \times \mathbb{R}^{+}
$$

describing 18 complex parameters and two real ones corresponding to the Kähler class of the fiber and the base.

In fact, the elliptic fibration reduces $\mathbf{S O}(3,19)$ to $\mathbf{S O}(2,18)$. Inspired by F-theory/ heterotic string duality in eight dimensions, the factor $\frac{\mathbf{S O}(2,18)}{\mathbf{S O}(2) \times \mathbf{S O}(18)}$ can be decomposed

$$
\frac{\mathbf{S O}(2,18)}{\mathbf{S O}(2) \times \mathbf{S O}(18)}=\frac{\mathbf{S O}(2,2)}{\mathbf{S O}(2) \times \mathbf{S O}(2)} \times \frac{\mathbf{S O}(2,16)}{\mathbf{S O}(2) \times \mathbf{S O}(16)}
$$

Similarity like in M-theory, the the factor $\frac{\mathbf{S O}(2,16)}{\mathbf{S O}(2) \times \mathbf{S O}(16)}$ can be reduced to two copies of $\frac{\mathbf{S O}(1,16)}{\mathbf{S O}(16)}$. Using the equivalence $\frac{\mathbf{S O}(2,2)}{\mathbf{S O}(2) \times \mathbf{S O}(2)} \sim \frac{\mathbf{S O}(2,1)}{\mathbf{S O}(2)} \times \frac{\mathbf{S O}(2,1)}{\mathbf{S O}(2)}$, the $\frac{\mathbf{S O}(2,18)}{\mathbf{S O}(2) \times \mathbf{S O}(18)}$ can be decomposed

$$
\frac{\mathbf{S O}(2,18)}{\mathbf{S O}(2) \times \mathbf{S O}(18)} \rightarrow \frac{\mathbf{S O}(2,1)}{\mathbf{S O}(2)} \times \frac{\mathbf{S O}(2,1)}{\mathbf{S O}(2)} \times \frac{\mathbf{S O}(1,16)}{\mathbf{S O}(16)}
$$

An inspection shows that the factor $\frac{\mathbf{S O}(2,1)}{\mathbf{S O}(2)} \times \frac{\mathbf{S O}(2,1)}{\mathbf{S O}(2)}$ correspond to $\mathbf{U}(1)^{4}$ gauge symmetry. From heterotic string point of view, these gauge fields can be classified in two categories. Indeed, $\mathrm{U}(1)^{2}$ are obtained from the ten dimensional metric $g_{\mu \nu}$, while the remaining ones come from the $B_{\mu \nu}$ field. As in M-theory compactification, $\frac{\mathrm{SO}(2,1)}{\mathrm{SO}(2)} \times \frac{\mathrm{SO}(2,1)}{\mathrm{SO}(2)}$ could be linked with two copies of 1-qubit. The last factor $\mathbf{S O}(1,16)$ corresponds $\mathbf{U}(1)^{16}$ gauge field associated with Cartan subalgebras of $E_{8} \times E_{8}$ (or $\mathbf{S O}(32)$ ) gauge symmetry. We expect that this factor can be related to a 4-qubit system. We believe that this connection deserves a deeper investigation.

Moreover, it will be interesting to consider the corresponding superqubit systems. This could be associated with supermanifold $T^{4, k}$ equipped with four bosonic coordinates and $k$ fermionic coordinates. It is recalled that supermanifolds have been investigated in connection with quantum superlogics [29]. A possible link can be done by fixing the value of $k$. For case of $k=4$, this can produce a K3 supersurface considered as a leading example of Calabi-Yau supermanifolds. The even and odd submanifolds can be associated with bosonic and fermionic invariant forms.

This work comes up with many open questions. One of them concerns connections with higher dimensional Calabi-Yau manifolds. It could also be interesting to look for qubit solutions from the string Calabi-Yau moduli space controlled by complex and Kähler deformations. Moreover, many concepts, which have been developed in quantum information theory including gates, circuits and entanglement, could have geometric representations. It should be of interest to study these issues in the context of the Hodge diagram analysis and physical theories related to black holes and branes. These issues will be addressed elsewhere.

Acknowledgments. AS is supported by FPA2012-35453. 


\section{References}

[1] H. Ooguri, A. Strominger, C. Vafa, Black Hole Attractors and the Topological String, Phys.Rev.D70(2004)106007, arXiv:hep-th/0405146.

[2] M. Guica, A. Strominger, Wrapped M2/M5 Duality, arXiv:hep-th/0701011.

[3] B. Haghighat, S. Murthy, C. Vafa, S. Vandoren, F-Theory, Spinning Black Holes and Multistring Branches, arXiv:1509.00455.

[4] S. Ferrara, R. Kallosh, A. Strominger, $N=2$ Extremal Black Holes, Phys. Rev. D52 (1995) 5412, hep-th/9508072.

[5] S. Ferrara and R. Kallosh, Supersymmetry and Attractors, Phys. Rev. D54 (1996) 1514, hep-th/9602136.

[6] R. Ahl Laamara, M. Asorey, A. Belhaj, A, Segui, Extremal Black Brane Attractors on The Elliptic Curve, J.Phys. A43 (2010) 105401, arXiv:0907.0093.

[7] L. Borsten, M. J. Duff, P. Lévay, The black-hole/qubit correspondence: an up-to-date review, arXiv:1206.3166.

[8] L. Borsten, M.J. Duff, A. Marrani, W. Rubens, On the Black-Hole/Qubit Correspondence, Eur.Phys.J.Plus 126 (2011) 37, arXiv:1101.3559.

[9] P. Lévay, Qubits from extra dimensions, Phys. Rev. D 84 (2001) 125020.

[10] A. Belhaj, M. B. Sedra, A. Segui, Graph Theory and Qubit Information Systems of Extremal Black Branes, J.Phys. A48 (2015) 045401, arXiv:1406.2578

[11] Y. Aadel, A. Belhaj, Z. Benslimane, M. B. Sedra, A. Segui, Qubits from Adinkra Graph Theory via Colored Toric Geometry, arXiv:1506.02523.

[12] A. Belhaj, H. Ez-Zahraouy, M. B. Sedra, Toric Geometry and String Theory Descriptions of Qudit Systems, J.Geom.Phys. 95 (2015) 21, arXiv:1408.3952

[13] M. B. Schulz, E. F. Tammaro, M-theory/type IIA duality and K3 in the Gibbons-Hawking approximation, arXiv:1206.1070.

[14] T. Okazaki, Membrane quantum mechanics, Nucl.Phys. B890 (2014) 400.

[15] P. S. Aspinwall, K3 Surfaces and String Duality, arXiv:hep-th/9611137.

[16] C. Vafa, Lectures on Strings and Dualities, arXiv:hep-th/9702201. 
[17] E. H. Saidi, A. Segui, Entropy of Pairs of Dual Attractors in six and seven Dimensions, arXiv:0803.2945 [hep-th].

[18] M. A. Nielsen, I. L. Chuang, Quantum Computation and Quantum Information Cambridge University Press, New York, NY, USA, 2000.

[19] D. R. Terno, Introduction to relativistic quantum information, arXiv:quant-ph/0508049.

[20] M. Kargarian, Entanglement properties of topological color codes, Phys. Rev. A78 (2008)062312, arXiv:0809.4276.

[21] M. Cvetic, G.W. Gibbons, C.N. Pope, Compactifications of Deformed Conifolds, Branes and the Geometry of Qubits, arXiv:1507.07585.

[22] M. Aganagic, D. Jafferis, N. Saulina, Branes, Black Holes and Topological Strings on Toric Calabi-Yau Manifolds, JHEP0612 (2006) 018, arXiv:hep-th/0512245.

[23] Vasily E. Tarasov, Quantum Computer with Mixed States and Four-Valued Logic Journal of Physics A. 25 (2002) 5207, quant-ph/0312131.

[24] S. Katz, P. Mayr, C. Vafa, Mirror symmetry and Exact Solution of $4 D$ N=2 Gauge Theories I, Adv.Theor.Math.Phys. 15(1998)53, arXiv:hep-th/9706110.

[25] C. Vafa, Evidence for F-theory, Nucl. Phys. B 469 (1996) 403, hep-th/9602022.

[26] V. K. Oikonomou, F-theory Yukawa Couplings and Supersymmetric Quantum Mechanics, Nucl.Phys. B856 (2011) 1, arXiv:1107.0497.

[27] V.K.Oikonomou, F-theory and the Witten Index, Nucl. Phys. B850(2011)273, arXiv:1103.1289.

[28] V. K. Oikonomou, Graded Geometric Structures Underlying F-Theory Related Defect Theories, arXiv:1303.2537.

[29] J. Kouneiher, N. Da Costa, Superlogic Manifolds and Geometric approach to Quantum Logic, arXiv:1505.00756. 